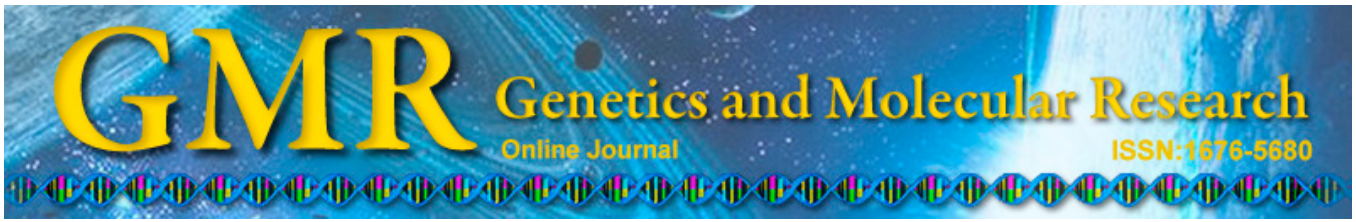

\title{
No difference of complete or incomplete left-sided malignant colonic obstruction on both short- and long-term outcomes
}

\author{
Z.-H. Liu*, C. Li*, N.-Q. Huang*, M.-J. Huang, H. Peng, L. Kang and \\ J.-P. Wang \\ Department of Colorectal Surgery, \\ Gastrointestinal Institute of Sun Yat-Sen University, \\ The Sixth Affiliated Hospital of Sun Yat-Sen University \\ (Guangdong Gastrointestinal Hospital), Guangzhou, Guangdong, China \\ *These authors contributed equally to this study. \\ Corresponding authors: J.-P. Wang / L. Kang \\ E-mail: cnzhdoc@126.com / ldrkang@163.com
}

Genet. Mol. Res. 13 (3): 7965-7978 (2014)

Received August 16, 2013

Accepted November 1, 2014

Published September 29, 2014

DOI http://dx.doi.org/10.4238/2014.September.29.10

\begin{abstract}
The impact of complete and incomplete colonic obstruction on the short- and long-term outcomes of malignant colorectal cancer has not yet been elucidated. The aim of this study was to investigate whether there was a difference in the impacts of the 2 types of obstruction on the short- and long-term outcomes of colorectal resection. This study included 224 colorectal cancer patients (162 patients with incomplete obstruction and 62 with complete obstruction) with left-sided malignant colonic obstruction who underwent surgical therapy between February 2007 and September 2012. The short- and long-term outcomes of surgical therapy were analyzed. No significant difference was found between the 2 groups with regard to short-term outcomes such as the curative resection rate $(80.86$ vs $70.97 \%, \mathrm{P}=$ $0.109)$, hospital stay time $(24.20 \pm 16.01$ vs $24.19 \pm 12.06, \mathrm{P}=0.999)$, and the overall and respective complications (32.72 vs 46.77\%, P =
\end{abstract}


0.051). Furthermore, no significant difference was found between the 2 groups with regard to long-term outcomes including the 1-, 3-, and 5 -year survival rates $(P=0.089)$, recurrence rates $(P=0.711)$, and recurrence-free survival rates $(\mathrm{P}=0.440)$. The 2 types of obstruction, i.e., complete and incomplete left-sided malignant colonic obstruction, had no impact on the short- and long-term outcomes of colorectal resection. Similar therapeutic methods can be used for treating both types of obstruction.

Key words: Left-sided malignant colonic obstruction; Complete obstruction; Incomplete obstruction

\section{INTRODUCTION}

It has been estimated that approximately 1 million new cases of colorectal cancer (CRC) with 500,000 CRC-related deaths occur annually (Ma et al., 2011; Siegel et al., 2012). Furthermore, the mortality and morbidity rates of CRC have been rising in recent years (Raine et al., 2010; Siegel et al., 2012); therefore, CRC represents the third most commonly diagnosed malignant carcinoma and is the fourth most frequent cause of mortality from malignant carcinoma (Liu et al., 2011). The overall incidence of CRC is high at 5\% in the general population, whereas the overall 5-year survival rate is less than $60 \%$ (Weitz et al., 2005). Furthermore, the lifetime risk of developing CRC is estimated to be up to $6 \%$ in normal people (Jemal et al., 2008). In fast developing countries like China, especially in Guangzhou, Beijing, Shanghai, and other major cities, the lifestyles of the general population have significantly altered, and therefore, CRC morbidity rates have rapidly increased over the recent decades (Sung et al., 2005; Zhang et al., 2012). Moreover, CRC presents as low rectal cancer in a high proportion of younger Chinese patients, and thus, it poses a serious threat to the Chinese population (Xu et al., 2010).

Most patients with CRC are not diagnosed until the disease is at an advanced stage with the presentation of intestinal obstruction or other emergency situations, owing to the absence of early typical symptoms and signs (Liu et al., 2011). Another reason may be that symptoms such as nausea, vomiting, and colicky abdominal pain are common and may be difficult to manage and cause significant physical distress and demoralization (Soriano and Davis, 2011). Approximately 7-47\% of patients with CRC may present colonic obstruction when diagnosed, with most of the patients presenting left-sided malignant colonic obstruction (LMCO) (Smothers et al., 2003; Coco et al., 2005), and in some patients, colonic obstruction may be the first symptom that prompts them to visit their doctor. Moreover, patients with an advanced stage of CRC are at a high risk of postoperative recurrence (Rahbari et al., 2012), which may cause colonic obstruction needing re-colectomy.

Although therapeutic management of LMCO includes hydration, nutrition, and endoscopic palliative options, surgical resection is still considered the best and final therapeutic option (Park et al., 2011). As per the guidelines set by the consensus conference of the world society of emergency surgery and peritoneum and surgery society (Ansaloni et al., 2010), when neoadjuvant therapy could be expected, loop colostomy and staged procedure should be adopted in case of a dramatic scenario. Hartmann's procedure should be performed in case of a high risk of anastomotic dehiscence. Subtotal and total colectomy should be attempted 
in case of cecal perforation or synchronous colonic neoplasm. Primary resection and anastomosis with manual decompression could be considered as the procedure of choice. Colonic stents represent the best option when skills are available. A recent randomized controlled trial indicated that colonic stenting has no decisive clinical advantages over emergency surgery, except that the emergency surgery could be changed to a selective procedure over the course of colonic stenting (van Hooft et al., 2011). Furthermore, to some extent, the change from emergency surgery to a selective one could cause a change from complete LMCO to incomplete LMCO, indirectly indicating that there is no difference in the outcomes between the surgery for complete LMCO and that for incomplete LMCO. However, as yet, there is no direct evidence for comparing the short- and long-term outcomes between the surgery for complete LMCO and that for incomplete LMCO.

Our previous study and other related studies have reported that differences were observed in the postoperative mortality rate, recurrence rate, 5-year survival rate, and proportion of liver metastasis between CRC patients with and those without obstruction (van Hooft et al., 2011; Yang et al., 2011). Colonic obstruction is an independent indicator of survival and postoperative recurrence in CRC patients, and patients with CRC obstruction have a worse overall survival rate with a high postoperative recurrence rate than do the control group patients (Yang et al., 2011). In our present study, we investigated the impact of complete and incomplete LMCO on the short- and long-term postoperative outcomes of colorectal resection, in order to provide more information on the treatment of the 2 types of LMCO.

\section{MATERIAL AND METHODS}

\section{Patients}

All patients who presented left-sided colonic obstruction and underwent colectomy between February 2007 and September 2012 at the Sixth Affiliated Hospital of Sun Yat-Sen University, formerly known as the Sixth People's Hospital of Guangzhou, were reviewed for inclusion in our study.

For definitive diagnosis before treatment, patients with suspected left-sided colonic obstruction underwent routine physical examination, laboratory tests, and imaging studies including 3-phase contrast-enhanced computed tomography (CT), magnetic resonance imaging (MRI), and colonoscopy. Thereafter, final diagnosis was made by comprehensive analysis of patient medical history, physical examination findings, abdominal CT scan results, colonoscopy features, and surgical findings as previously described (Yang et al., 2011). Death occurring within 30 days after the colectomy was referred to as postoperative mortality, and cases of postoperative mortality were excluded from the survival analysis (Yang et al., 2011). All causes of death were included in the study, and the overall survival rates at 1,3 , and 5 years were considered as the crude survival rates. The FOLFOX regimen was considered the firstline regimen for adjuvant chemotherapy in patients with TNM stages II and III within 1 month after the surgery was performed. Curative surgery was performed when there was complete resection of tumor with no local or distant residual malignancy at the completion of the procedure, whereas palliative procedure was performed when there was residual malignancy or metastasis present after the completion of the operation (Yang et al., 2011).

The characteristics and pathological findings of the patients were recorded (Table 1), including age, gender, body mass index (BMI), site of CRC (descending colon, sigmoid, or 
rectum), chemotherapy, length to anus edge, tumor size, tumor movement, macroscopic type (polyploidy, ulcerative, or infiltrative), depth of invasion [T1: The cancer grows through the muscularis mucosa and extends into the submucosa; T2: The cancer grows through the submucosa and extends into the muscularis propria (thick outer muscle layer); T3: The cancer grows through the muscularis propria and extends into the outermost layers of the colon or rectum, but not through them. It does not reach any nearby organs or tissues; or T4: The cancer grows through the serosa or through the wall of the colon or rectum and attaches to or invades into nearby tissues or organs], nodal involvement, liver metastasis, surgical approach, histological grade (well, moderately, or poorly differentiated), TNM stage, histological type (adenocarcinoma, mucinous adenocarcinoma, or signet ring cell tumor), peritoneal carcinomatosis, surgical approach (laparoscopy or not), and tumor biomarkers (carcinoembryonic antigen, carbohydrate antigen 19-9, carbohydrate antigen 125, carbohydrate antigen 153, and $\alpha$-fetoprotein). Furthermore, evaluation variables were recorded (Table 2), which included the curative resection rate; hospital stay time; 1-, 3-, and 5-year survival rates, recurrence rates, and recurrence-free survival rates; and complications such as death, peritonitis, pneumonia, urinary tract infection (UTI), incisional infection, intra-abdominal hemorrhage, intraintestinal hemorrhage, uroschesis, deep vein thrombosis (DVT), postoperative intestinal obstruction, ureteral injury, anastomotic leakage, stoma complications (stenosis, necrosis, exodus, retraction, and hematoma), autonomic function recovery time ( $<7$ days, 7-14 days, $>14$ days), and sexual dysfunction. This study was conducted in accordance with the Declaration of Helsinki and was approved by the Ethics Committee of Sun Yat-Sen University. Written informed consent was obtained from all the participants in the study.

\section{Inclusion and exclusion criteria}

The inclusion criteria were as follows: a) Age $>18$ years; b) Presentation of obstructive symptoms; c) Dilation of the colon; d) CT scan, MRI, or colonoscopy findings suggesting left-sided malignant obstruction; e) Histological determination of adenocarcinoma.

The exclusion criteria were as follows: a) Obstruction located proximal to the splenic flexure or distal to the rectosigmoid junction, with symptoms suggesting bowel perforation; b) Obstruction caused by a noncolonic malignancy or a benign disease; c) Septic symptoms; d) Spontaneous pneumoperitoneum; e) Adjacent small bowel involvement; f) Patients with preoperative stage VI; g) Age $<18$ years; h) Pregnancy.

\section{Surgical technique}

As previously described (Hemandas et al., 2010), open colectomy was performed using a midline incision, while laparoscopy was performed using a 5-port technique and a transverse incision of approximately $5 \mathrm{~cm}$ for extraction of the specimen. Patients were placed in the improved lithotomy position (Sachs and Peters, 1999) with the surgeon standing on the right side of the patient. Clips or vascular staplers were used for mobilization, isolation, and ligation of the main vessels from the medial to the lateral aspect of colorectal tissues (Mehmood et al., 2011). As previously described, total mesorectal excision was performed using monopolar diathermy for rectal cancers (Heald et al., 1982; Hemandas et al., 2010). A wound protector was used to protect the sample extraction site, and anastomoses were performed intracorporeally. A loop ileostomy was routinely planted for mid and low rectal 
anastomoses for postoperative recovery of anastomotic stoma. Postoperative pain control was selectively performed using epidural catheters and patient-controlled analgesia.

Patient care was carefully managed postoperatively, and the patients were allowed home when the discharge criteria were met (Kehlet and Wilmore, 2002). Oral bowel preparation and nasogastric tubes were routinely used. Moreover, minimal use of drains, early unrestricted postoperative oral intake of fluids and light diet, early mobilization, selected use of epidural catheters, and restricted use of narcotics were allowed (Hemandas et al., 2010). Preoperative carbohydrate loading was not used.

\section{Follow-up}

Follow-up was performed by 1 commissioner (Yi BY) and 3 surgeons (Huang MJ, Peng $\mathrm{H}$, and Zhang XW), and it included physical examination, hematological-biochemical examinations, serum carcinoembryonic antigen level assay analysis, chest radiography, and abdominal and/or pelvic CT scans every 3 months during the first year, every 6 months during the subsequent 2 years, and then once a year, as previously described (Yang et al., 2011). Follow-up was performed through clinic appointments, home visits, or letters/phone calls to update information constantly. The follow-up end point was September 2012.

\section{Statistical analysis}

Statistical analysis was performed using the program SPSS for Windows, version 17.0 (SPSS, Chicago, IL, USA). Demographic data for the 2 groups were compared using the Student $t$-test for continuous data and the $\chi^{2}$ test for categorical data. The survival rate was calculated using the Kaplan-Meier method, and the differences in survival were compared using the log-rank test. The differences between the 2 groups were considered to be statistically significant if the $\mathrm{P}$ value was $<0.05$. Our statistical analysis data were checked by a statistical analyst.

\section{RESULTS}

\section{General information}

Three hundred and twenty-eight patients with CRC were included in our study after a review of patients who presented left-sided colonic obstruction and underwent surgical therapy between February 2007 and September 2012 at our hospital. Among these patients, 206 consecutive patients had low preoperative serum albumin levels, whereas 122 consecutive patients had normal preoperative serum albumin levels. Of patients with incomplete LMCO and those with complete LMCO, 165 and 64 patients, respectively, met the inclusion criteria. However, 3 patients from the incomplete LMCO group and 2 patients from the complete LMCO group died during the follow-up period. Therefore, 224 patients, including 162 patients $(72.32 \%)$ with incomplete obstruction and $62(27.68 \%)$ with complete obstruction, were finally included in the study (Table 1). The results show that no significant difference was observed in the baseline patient characteristics between the 2 patient groups, i.e., the complete and incomplete LMCO groups, with respect to factors such as age, gender, BMI, chemotherapy, length to anus edge, tumor size, tumor movement, macroscopic type, depth of invasion, nodal involvement, liver metastasis, surgical approach, histological grade, TNM 
stage, histological type, peritoneal carcinomatosis, disease recurrence, and tumor biomarkers (carcinoembryonic antigen, carbohydrate antigen 19-9, carbohydrate antigen 125, carbohydrate antigen 153 , and $\alpha$-fetoprotein).

Table 1. Baseline characteristics of patients with left-sided malignant colonic obstruction included in our study.

\begin{tabular}{|c|c|c|c|c|}
\hline & \multicolumn{2}{|c|}{ Preoperative serum albumin level } & \multirow[t]{2}{*}{$\chi^{2 / t}$ value } & \multirow[t]{2}{*}{ P value } \\
\hline & Normal & Recurrence & & \\
\hline Number & 162 & 62 & & \\
\hline Age (years) & $61.12 \pm 15.26$ & $58.61 \pm 14.29$ & 0.847 & 0.398 \\
\hline Gender (all) & & & 1.775 & 0.183 \\
\hline Male & 94 & 42 & & \\
\hline Female & 68 & 20 & & \\
\hline Age group years & & & 1.491 & 0.475 \\
\hline$\leq 40$ & 20 & 6 & & \\
\hline $41-64$ & 64 & 30 & & \\
\hline$\geq 65$ & 78 & 26 & & \\
\hline Body mass index & $21.48 \pm 3.50$ & $22.45 \pm 3.53$ & 1.235 & 0.219 \\
\hline Site of colorectal carcinoma & & & 2.081 & 0.353 \\
\hline Decending colon & 45 & 20 & & \\
\hline Sigmoid & 32 & 16 & & \\
\hline Rectum & 85 & 26 & & \\
\hline Chemotherapy & $145 / 162$ & $60 / 62$ & 3.041 & 0.081 \\
\hline Length to anus edge & $23.88 \pm 24.18$ & $34.44 \pm 25.22$ & 1.613 & 0.109 \\
\hline Tumor size & & & 0.566 & 0.452 \\
\hline$\leq 5$ & 101 & 42 & & \\
\hline$>5$ & 61 & 20 & & \\
\hline Tumor movement & $12 / 162$ & $4 / 62$ & 0.062 & 0.804 \\
\hline Macroscopic type & & & 3.892 & 0.143 \\
\hline Polyploid & 77 & 32 & & \\
\hline Ulcerative & 75 & 22 & & \\
\hline Infiltrative & 10 & 8 & & \\
\hline Depth of invasion & & & 2.413 & 0.491 \\
\hline $\mathrm{T} 1$ & 7 & 1 & & \\
\hline $\mathrm{T} 2$ & 11 & 2 & & \\
\hline $\mathrm{T} 3$ & 103 & 43 & & \\
\hline $\mathrm{T} 4$ & 41 & 16 & & \\
\hline Nodes involvement & & & 5.786 & 0.055 \\
\hline N0 & 82 & 22 & & \\
\hline N1 & 45 & 30 & & \\
\hline N2 & 35 & 10 & & \\
\hline Liver metastasis & $27 / 162$ & $6 / 62$ & 1.744 & 0.187 \\
\hline Laparoscopy colorectomy & $46 / 162$ & $18 / 62$ & 0.009 & 0.925 \\
\hline Histological grade & & & 3.134 & 0.209 \\
\hline Well differentiated & 63 & 18 & & \\
\hline Moderately differentiated & 80 & 32 & & \\
\hline Poorly differentiated & 19 & 12 & & \\
\hline TNM stage & & & 5.649 & 0.130 \\
\hline I & 17 & 2 & & \\
\hline II & 55 & 20 & & \\
\hline III & 51 & 28 & & \\
\hline IV & 39 & 12 & & \\
\hline Histological type & & & 0.510 & 0.775 \\
\hline Adenocarcinoma & 139 & 55 & & \\
\hline Mucinous adenocarcinoma & 21 & 6 & & \\
\hline Signet ring cell tumor & 2 & 1 & & \\
\hline Peritoneal carcinomatosis & $10 / 162$ & $6 / 62$ & 0.386 & 0.534 \\
\hline \multicolumn{5}{|l|}{ Tumor biomarkers } \\
\hline Carcinoembryonic antigen & $24.59 \pm 67.68$ & $16.48 \pm 33.92$ & 0.617 & 0.538 \\
\hline Carbohydrate antigen $19-9$ & $93.88 \pm 481.66$ & $3842.78 \pm 23198.42$ & 1.754 & 0.081 \\
\hline Carbohydrate antigen 125 & $17.41 \pm 22.19$ & $26.02 \pm 31.16$ & 1.966 & 0.051 \\
\hline Carbohydrate antigen 153 & $6.85 \pm 5.84$ & $5.08 \pm 4.56$ & 1.152 & 0.252 \\
\hline$\alpha$-fetoprotein & $11.53 \pm 87.08$ & $3.65 \pm 4.34$ & 0.383 & 0.1703 \\
\hline
\end{tabular}




\section{Short-term outcomes}

Short-term outcome analysis showed no significant differences between the incomplete and complete LMCO groups, with regard to the curative resection rates, hospital stay time, and complications such as death, peritonitis, pneumonia, UTI, incisional infection, intraabdominal hemorrhage, intraintestinal hemorrhage, uroschesis, DVT, postoperative intestinal obstruction, ureteral injury, anastomotic leakage, stoma complications, autonomic function recovery time, and sexual dysfunction. The curative resection rate was $80.86 \%(131 / 162)$ in the incomplete LMCO group, whereas it was $70.97 \%(44 / 62)$ in the complete LMCO group ( $\mathrm{P}>0.05$; Table 2). The hospital stay time was $24.20 \pm 16.01$ days for the incomplete LMCO group, whereas it was $24.19 \pm 12.06$ days for the complete LMCO group $(\mathrm{P}>0.05$; Table 2 ). No significant differences in the overall complication rates were observed between the 2 groups, with $32.72 \%$ (53/162) overall complication rate in the incomplete LMCO group and $46.77 \%(29 / 62)$ in the complete LMCO group (P > 0.05; Table 2). Moreover, there were no significant differences between the 2 groups with respect to the main complications including peritonitis (1/162 vs 1/62), pneumonia (4/162 vs 2/62), UTI (6/162 vs 4/62), incisional infection $(9 / 162 v s 6 / 62)$, intra-abdominal hemorrhage (2/162 vs 1/62), intraintestinal hemorrhage (1/162 vs 1/62), uroschesis (9/162 vs 2/62), DVT (3/162 vs 2/62), postoperative intestinal obstruction (8/162 vs 2/62), ureteral injury (1/162 vs $0 / 62)$, anastomotic leakage (5/162 vs $1 / 62)$, stoma complications such as stenosis $(0 / 162$ vs $1 / 62)$, necrosis $(1 / 162 v s 0 / 62)$, exodus $(1 / 162$ vs $0 / 62)$, retraction $(2 / 162 v s 1 / 62)$, and hematoma (1/162 vs $0 / 62)$, autonomic function recovery time ( $<7$ days/7-14 days/ $>14$ days, $150 / 8 / 4$ vs 55/5/2), and sexual dysfunction (31/162 vs $8 / 62$, all $\mathrm{P}>0.05$ ). One patient from the incomplete LMCO group died from multiorgan dysfunction syndrome in the hospital, while another patient from the complete LMCO group died from postoperative pneumonia. No ureteral injuries were observed in the complete LMCO group. In the incomplete LMCO group, 2 patients developed 3 complications simultaneously, while 6 patients developed 2 complications simultaneously. In the complete LMCO group, 1 patient developed 5 complications simultaneously (pneumonia, UTI, uroschesis, postoperative intestinal obstruction, and sexual dysfunction), another patient developed 3 complications simultaneously, and 5 patients developed 2 complications simultaneously. Patients with peritonitis were given conservative treatment in the incomplete LMCO group and surgical therapy in the complete LMCO group. Four patients from each group developed postoperative pneumonia. Six patients in the incomplete LMCO group and 4 patients in the complete LMCO group developed UTI. Two patients in the incomplete LMCO group and 1 patient in the complete LMCO group developed intra-abdominal hemorrhage, while 1 patient in the incomplete LMCO group and 1 patient in the complete LMCO group developed intraintestinal hemorrhage. Three patients in the incomplete LMCO group and 2 patients in the complete LMCO group developed deep vein thrombosis. One patient in the incomplete LMCO group developed ureteral injury. In the incomplete LMCO group, 5 patients with postoperative intestinal obstruction were given conservative treatment, whereas 2 were given surgical therapy, and 3 patients with anastomotic leakage were given surgical therapy, whereas 2 were given conservative treatment. In the complete LMCO group, 2 patients with postoperative intestinal obstruction were given conservative treatment, whereas 1 was given surgical therapy, and 1 patient with anastomotic leakage was given conservative treatment. In both groups, patients with stoma complications were given conservative treatment and were released. 
Table 2. Short- and long-term outcomes after colorectomy of patients with the left-sided colorectal cancer and complete or incomplete obstruction.

\begin{tabular}{|c|c|c|c|c|}
\hline & \multicolumn{2}{|c|}{ Preoperative serum albumin level } & \multirow[t]{2}{*}{$\chi^{2} / t$ value } & \multirow[t]{2}{*}{$P$ value } \\
\hline & Incomplete & Complete & & \\
\hline Curative resection rate & $131 / 162$ & $44 / 62$ & 2.570 & 0.109 \\
\hline Hospital stay time (days) & $24.20 \pm 16.01$ & $24.19 \pm 12.06$ & 0.001 & 0.999 \\
\hline \multicolumn{5}{|l|}{ Survival rate } \\
\hline 1 year & $143 / 144$ & $56 / 58$ & 0.674 & 0.412 \\
\hline 3 years & $58 / 129$ & $18 / 42$ & 0.057 & 0.812 \\
\hline 5 years & $24 / 72$ & $8 / 36$ & 0.711 & 0.399 \\
\hline \multicolumn{5}{|l|}{ Recurrence rate } \\
\hline 1 year & $8 / 144$ & $3 / 58$ & 0.048 & 0.827 \\
\hline 3 years & $26 / 129$ & $10 / 42$ & 0.255 & 0.614 \\
\hline 5 years & $41 / 72$ & $19 / 36$ & 0.169 & 0.681 \\
\hline \multicolumn{5}{|l|}{ Recurrence-free survival rate } \\
\hline 1 year & $136 / 144$ & $55 / 58$ & 0.048 & 0.827 \\
\hline 3 years & $103 / 129$ & $32 / 42$ & 0.255 & 0.614 \\
\hline 5 years & $31 / 72$ & $17 / 36$ & 0.169 & 0.681 \\
\hline Complications (all) & $53 / 162$ & $29 / 62$ & 3.819 & 0.051 \\
\hline Death & $1 / 162$ & $1 / 62$ & 0.502 & 0.478 \\
\hline Peritonitis & $1 / 162$ & $1 / 62$ & 0.502 & 0.478 \\
\hline Pneumonia & $4 / 162$ & $4 / 62$ & 1.070 & 0.301 \\
\hline Urinary tract infection & $6 / 162$ & $4 / 62$ & 0.280 & 0.597 \\
\hline Incisional infection & $9 / 162$ & $6 / 62$ & 0.649 & 0.421 \\
\hline Intra-abdominal hemorrhage & $2 / 162$ & $1 / 62$ & 0.049 & 0.826 \\
\hline Intra-intestine hemorrhage & $1 / 162$ & $1 / 62$ & 0.502 & 0.478 \\
\hline Uroschesis & $9 / 162$ & $2 / 62$ & 0.116 & 0.733 \\
\hline Deep vein thrombosis & $3 / 162$ & $2 / 62$ & 1.273 & 0.259 \\
\hline Postoperative intestinal obstruction & $8 / 162$ & $2 / 62$ & 0.038 & 0.846 \\
\hline Ureteral injury & $1 / 162$ & $0 / 62$ & 0.384 & 0.535 \\
\hline Anastomotic leakage & $5 / 162$ & $1 / 62$ & 0.022 & 0.882 \\
\hline Stoma complications & $5 / 162$ & $2 / 62$ & 0.003 & 0.957 \\
\hline Stenosis & $0 / 162$ & $1 / 62$ & 0.382 & 0.536 \\
\hline Necrosis & $1 / 162$ & $0 / 62$ & 0.384 & 0.535 \\
\hline Exodus & $1 / 162$ & $0 / 62$ & 0.384 & 0.535 \\
\hline Retraction & $2 / 162$ & $1 / 62$ & 0.049 & 0.826 \\
\hline Hematoma & $1 / 162$ & $0 / 62$ & 0.384 & 0.535 \\
\hline Autonomic function recovery time & & & 0.873 & 0.646 \\
\hline Less than 7 days & 150162 & $55 / 62$ & & \\
\hline $7-14$ days & $8 / 162$ & $5 / 62$ & & \\
\hline More than 14 days & $4 / 162$ & $2 / 62$ & & \\
\hline Sexual dysfunction & $31 / 162$ & $8 / 62$ & 1.211 & 0.271 \\
\hline
\end{tabular}

\section{Long-term outcomes}

Long-term outcome analysis showed no significant differences between the complete and incomplete LMCO groups with respect to the 1-, 3-, and 5-year survival rates, recurrence rates, and recurrence-free survival rates. The mean follow-up period was $35 \pm 19$ months in the incomplete LMCO group and $33 \pm 18$ months in the complete LMCO group. During the follow-up period, 26 patients $(16.05 \%, 26 / 162)$ in the incomplete LMCO group and 14 patients $(22.58 \%, 14 / 62)$ in the complete LMCO group died. In the incomplete LMCO group, 25 of the 26 patients died of cancer and 1 patient died of respiratory failure, whereas in the complete LMCO group, all of the 14 patients died of cancer. The survival rates at 1,3 , and 5 years were 99.31\% (143/144), 44.96\% (58/129), and 33.33\% (24/72), respectively, in the incomplete LMCO group, and were $96.55 \%$ (56/58), 42.86\% (18/42), and 22.22\% (8/36), respectively, in the complete LMCO group. There was no significant difference in the survival rates between 
the 2 patient groups $\left(\chi^{2}=2.900, \mathrm{P}=0.089\right.$; Figure 1$)$, and in the survival rates at 1,3 , and 5 years, respectively (all $\mathrm{P}>0.05$; Table 2 ). The recurrence rates at 1,3 , and 5 years were $5.56 \%$ (8/144), $20.16 \%$ (26/129), and 56.94\% (41/72), respectively, in the incomplete LMCO group, and were $5.17 \%(3 / 58), 23.81 \%(10 / 42)$, and $52.78 \%(19 / 36)$, respectively, in the complete LMCO group. There was no significant difference in the recurrence rates between the 2 patient groups $\left(\chi^{2}=0.138, P=0.711\right.$; Figure 2$)$, and in the recurrence rates at 1,3 , and 5 years, respectively (all $\mathrm{P}>0.05$; Table 2 ). The recurrence-free survival rates at 1,3 , and 5 years were $94.44 \%$ (136/144), $79.84 \%(103 / 129)$, and $43.06 \%$ (41/72), respectively, in the incomplete LMCO group, and were $94.83 \%$ (3/58), 76.19\% (10/42), and 47.22\% (19/36), respectively, in the complete LMCO group. There was no significant difference in the recurrence-free survival rates between the 2 patient groups $\left(\chi^{2}=0.595, \mathrm{P}=0.440\right.$; Figure 3$)$, and in the recurrence-free survival rates at 1,3 , and 5 years, respectively (all $\mathrm{P}>0.05$; Table 2 ).

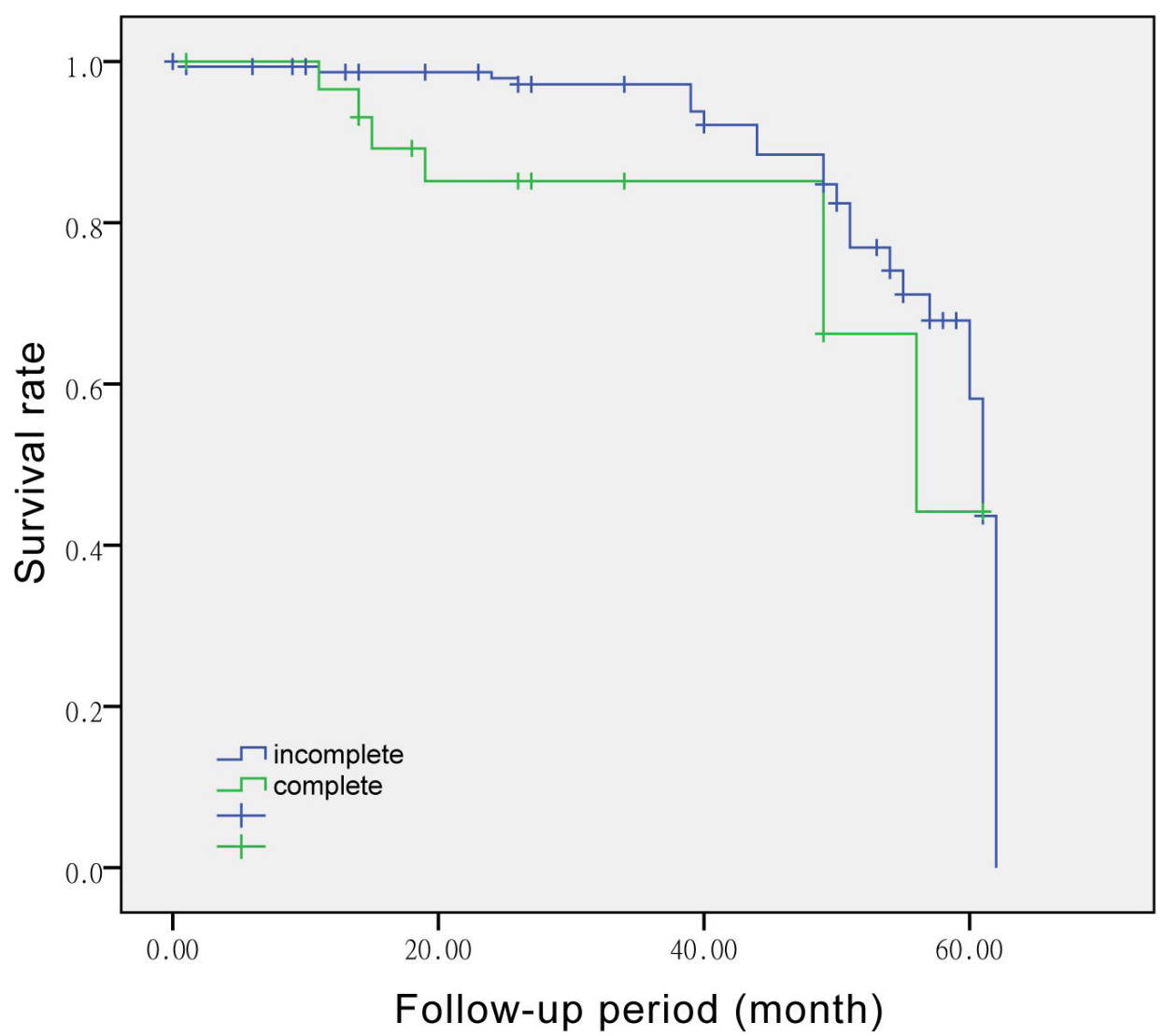

Figure 1. Kaplan-Meier curve shows overall survival rates for patients with normal or low preoperative serum albumin level who underwent colorectomy. Result showed no significant difference in survival rates between the two patient groups $\left(\chi^{2}=1.765 ; \mathrm{P}=0.184\right)$. Normal $=$ normal preoperative serum albumin level group; low $=$ low preoperative serum albumin level group. 


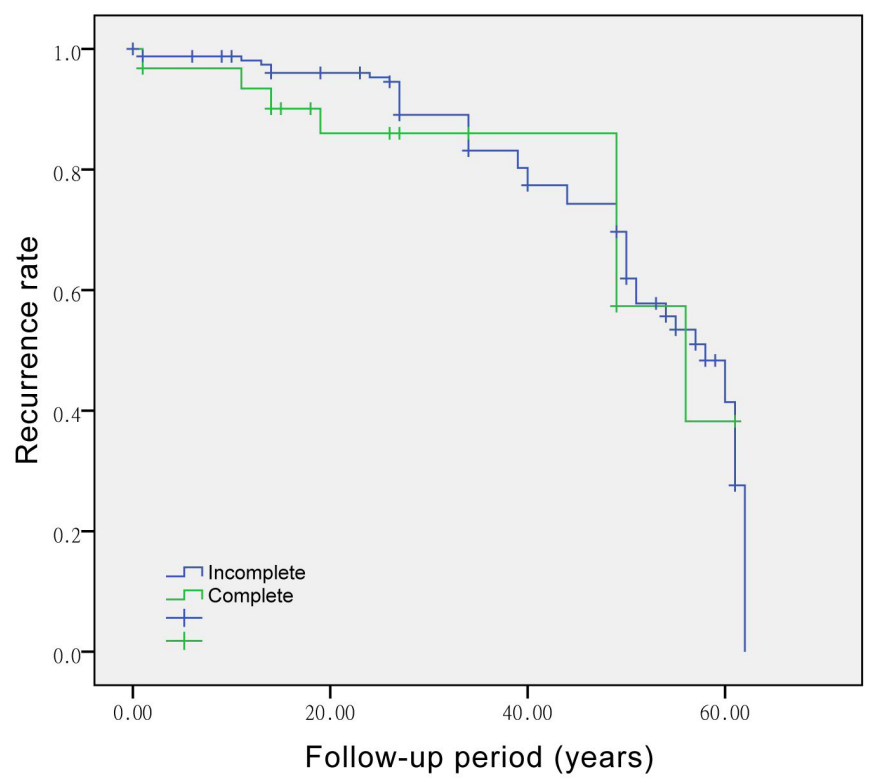

Figure 2. Kaplan-Meier curve shows recurrence rates for patients with normal or low preoperative serum albumin level who underwent colorectomy. Result showed no significant difference in recurrence rates between the two patient groups $\left(\chi^{2}=0.203 ; \mathrm{P}=0.652\right)$. Normal $=$ normal preoperative serum albumin level group; low $=$ low preoperative serum albumin level group.

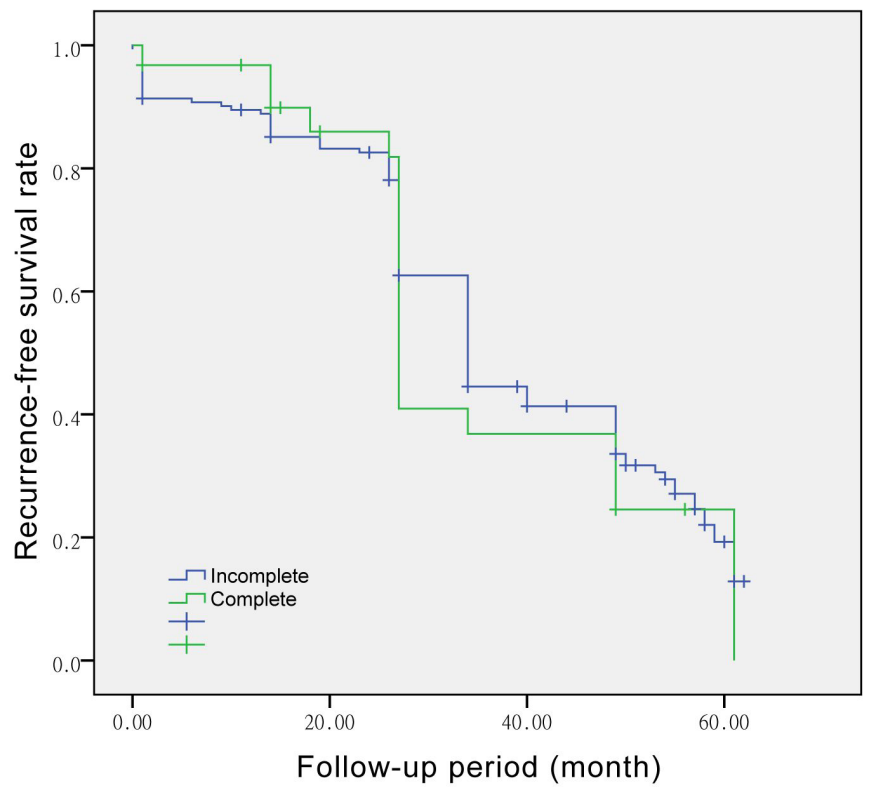

Figure 3. Kaplan-Meier curve shows overall recurrence-free survival rates for patients with normal or low preoperative serum albumin level who underwent colorectomy. Result showed no significant difference in recurrence-free survival rates between the two patient groups $\left(\chi^{2}=0.000 ; \mathrm{P}=0.993\right)$. Normal $=$ normal preoperative serum albumin level group; low $=$ low preoperative serum albumin level group. 


\section{DISCUSSION}

In recent years, with the rise in the mortality and morbidity rates of CRC, malignant colonic obstruction has been causing more complicated clinical problems in CRC patients (Jimenez-Perez et al., 2011; Song and Baron, 2011; van Hooft et al., 2011). Surgical colectomy is still considered the best and final choice for the treatment of LMCO (Song and Baron, 2011; van Hooft et al., 2011). Different short- and long-term outcomes related to the postoperative mortality rate, recurrence rate, 5 -year survival rate, and proportion of liver metastasis have been reported in CRC patients with and those without obstruction (van Hooft et al., 2011; Yang et al., 2011). However, to our knowledge, the different impacts of complete and incomplete LMCO on the short- and long-term postoperative outcomes have not yet been investigated. Moreover, it is unclear whether different therapeutic methods should be used for treating the 2 types of obstruction if the short- and long-term outcomes of complete and incomplete LMCO surgery differ. Since the establishment of our hospital 5 years ago, we have been interested in the study of the diagnosis and treatment of LMCO and have attempted to gain further information on the treatment of LMCO. In our present study, we investigated the impact of complete and incomplete LMCO on the short- and long-term postoperative outcomes in patients.

Between February 2007 and September 2012, patients who met the inclusion criteria mentioned above were included in this study, and no significant difference was found between their baseline characteristics. As shown in the results, the curative resection rates did not significantly differ between the complete and incomplete LMCO patient groups. This may be owing to the fact that the surgeons in our hospital can achieve R0 resection and colorectal mesenteric resection irrespective of whether the LMCO is complete or incomplete (Hemandas et al., 2010). Moreover, other indicators of short-term outcomes after LMCO surgery did not significantly differ between the incomplete and complete LMCO patient groups. The hospital stay times also did not significantly differ between the 2 groups, suggesting that postoperative recovery was affected mainly by the CRC itself, rather than by the type of obstruction (Yang et al., 2011). One patient in the incomplete LMCO group died from acute renal failure and multiorgan dysfunction syndrome caused by postoperative pneumonia and UTI, while another patient died from postoperative pneumonia because of a prolonged history of smoking. The patient with peritonitis in the incomplete LMCO group was given conservative treatment with antibiotics, whereas the one in the complete LMCO group was given surgical and adjuvant antibiotic therapy. In each of the 2 groups, 4 patients developed postoperative pneumonia, and all of these patients were treated with conservative therapy with antibiotics; 2 of these patients, 1 from each group, required ventilator support. Six patients in the incomplete LMCO group and 4 patients in the complete LMCO group developed UTI, and all of these patients were treated with conservative therapy with antibiotics. Two patients in the incomplete LMCO group and 1 patient in the complete LMCO group developed intra-abdominal hemorrhage, while 1 patient in the incomplete LMCO group and 1 patient in the complete LMCO group developed intraintestinal hemorrhage; all of these patients were treated using the surgical approach. Three patients in the incomplete LMCO group and 2 patients in the complete LMCO group developed deep vein thrombosis and received anticoagulant and thrombolysis therapy. Early activity of the postoperative lower extremity was recommended in all of these patients, and none of the other patients de- 
veloped thrombosis. Hemostatic medicine was not regularly used. One patient with ureteral injury in the incomplete LMCO group received surgical treatment and recovered. However, these indicators of short- and long-term outcomes did not differ significantly between the 2 groups. Seven patients with postoperative intestinal obstruction were given conservative treatment, whereas 3 patients were given surgical therapy; moreover, 3 patients with anastomotic leakage were given surgical therapy, whereas 3 patients were given conservative treatment. Stoma complications were also similar in patients. In the complete LMCO group, 1 patient presented stenosis, whereas in the incomplete LMCO group, 1 patient presented necrosis, 1 presented exodus, and 1 presented hematoma. Two retractions occurred in the incomplete LMCO group, and 1 in the complete LMCO group. All patients with stoma complications were given conservative treatment and were released. The overall rate of stoma complications was low because our postoperative nursing techniques were excellent, and the first and biggest stoma therapeutic school was established in China by our team. The 2 patient groups also showed similar postoperative sexual dysfunction rates and autonomic function recovery times. Above all, the main complications of short-term outcomes, including curative resection rates, hospital stay times, and complications such as death, peritonitis, pneumonia, UTI, incisional infection, intra-abdominal hemorrhage, intraintestinal hemorrhage, uroschesis, DVT, postoperative intestinal obstruction, ureteral injury, anastomotic leakage, stoma complications, autonomic function recovery times, and sexual dysfunction, did not significantly differ between the 2 patient groups.

No significant difference was found in the survival, recurrence, and recurrence-free survival rates, and the 1-, 3-, and 5-year survival, recurrence, and recurrence-free survival rates, respectively, indicating that the long-term outcomes may be associated with the patient characteristics themselves and the surgical treatment approach, rather than the types of obstruction. Moreover, CRC recurrence is still the most influencing factor of prognosis, because only 2 of the 40 patients did not die of cancer, but of respiratory failure or multiorgan dysfunction syndrome. No difference in the short- and long-term outcomes was found between the 2 patient groups, and therefore, it may not be necessary to differentiate between the therapeutic methods for LMCO treatment, except in the case of emergency treatment for obstruction, such as emergency surgery.

One limitation of our study is that we did not distinguish between emergency surgery and selective operation because it has recently been reported that selective surgery has no clinical advantages over emergency surgery, because conversion of an emergency surgery to a selective one did not show any advantages (van Hooft et al., 2011). The other limitation is related to the preoperative tumor biomarkers used in this study. The level of carbohydrate antigen 19-9 ranged from the normal level of approximately 20 to a highest value of $>30,000$. The carbohydrate antigen 19-9 level was $93.88 \pm 481.66$ in the incomplete LMCO group, whereas it was $3842.78 \pm 23198.42$ in the complete LMCO group. However, there was no significant difference in the values between the 2 groups $\left(\chi^{2}=1.754, \mathrm{P}=0.081\right.$; Table 1$)$.

\section{CONCLUSION}

The 2 types of obstruction, i.e., complete and incomplete LMCO, had no impact on the short- and long-term outcomes after colorectal resection. Similar therapeutic methods can be used for treating both complete and incomplete LMCO. 


\section{Conflicts of interest}

All authors declare no conflict of interest.

\section{ACKNOWLEDGMENTS}

The authors thank the medical staff at the Sixth Affiliated Hospital of Sun Yat-Sen University for their technical assistance during this study. Research supported by the National Natural Science Foundation of China (\#81100255 and \#81370480), and the foundation of New Star of Zhujiang Science and Technology Foundation (\#2013J2200023).

\section{REFERENCES}

Ansaloni L, Andersson RE, Bazzoli F, Catena F, et al. (2010). Guidelines in the management of obstructing cancer of the left colon: consensus conference of the world society of emergency surgery (WSES) and peritoneum and surgery (PnS) society. World J. Emerg. Surg. 5: 29.

Coco C, Verbo A, Manno A, Mattana C, et al. (2005). Impact of emergency surgery in the outcome of rectal and left colon carcinoma. World J. Surg. 29: 1458-1464.

Heald RJ, Husband EM and Ryall RD (1982). The mesorectum in rectal cancer surgery - the clue to pelvic recurrence? Br. J. Surg. 69: 613-616.

Hemandas AK, Abdelrahman T, Flashman KG, Skull AJ, et al. (2010). Laparoscopic colorectal surgery produces better outcomes for high risk cancer patients compared to open surgery. Ann. Surg. 252: 84-89.

Jemal A, Siegel R, Ward E, Hao Y, et al. (2008). Cancer statistics, 2008. CA Cancer J. Clin. 58: 71-96.

Jimenez-Perez J, Casellas J, Garcia-Cano J, Vandervoort J, et al. (2011). Colonic stenting as a bridge to surgery in malignant large-bowel obstruction: a report from two large multinational registries. Am. J. Gastroenterol. 106: 21742180 .

Kehlet H and Wilmore DW (2002). Multimodal strategies to improve surgical outcome. Am. J. Surg. 183: 630-641.

Liu Z, Qin H, Yang Z, Xia Y, et al. (2011). Randomised clinical trial: the effects of perioperative probiotic treatment on barrier function and post-operative infectious complications in colorectal cancer surgery - a double-blind study. Aliment. Pharmacol. Ther. 33: 50-63.

Ma Y, Zhang P, Wang F, Yang J, et al. (2011). Association between vitamin D and risk of colorectal cancer: a systematic review of prospective studies. J. Clin. Oncol. 29: 3775-3782.

Mehmood S, Ullah S, Hunter IA, Hartley JE, et al. (2011). Short-term outcomes of laparoscopic versus open colorectal cancer surgery. Ann. Surg. 254: 177-178.

Park JS, Choi GS, Jun SH, Hasegawa S, et al. (2011). Laparoscopic versus open intersphincteric resection and coloanal anastomosis for low rectal cancer: intermediate-term oncologic outcomes. Ann. Surg. 254: 941-946.

Rahbari NN, Bork U, Motschall E, Thorlund K, et al. (2012). Molecular detection of tumor cells in regional lymph nodes is associated with disease recurrence and poor survival in node-negative colorectal cancer: a systematic review and meta-analysis. J. Clin. Oncol. 30: 60-70.

Raine R, Wong W, Scholes S, Ashton C, et al. (2010). Social variations in access to hospital care for patients with colorectal, breast, and lung cancer between 1999 and 2006: retrospective analysis of hospital episode statistics. BMJ 340: b5479.

Sachs M and Peters J (1999). History of surgical instruments. 9. Surgical instruments and development of surgical technique of lithotomy incision. Zentralbl. Chir. 124: 1059-1066.

Siegel R, Naishadham D and Jemal A (2012). Cancer statistics, 2012. CA Cancer J. Clin. 62: 10-29.

Smothers L, Hynan L, Fleming J, Turnage R, et al. (2003). Emergency surgery for colon carcinoma. Dis. Colon Rectum 46: 24-30.

Song LM and Baron TH (2011). Stenting for acute malignant colonic obstruction: a bridge to nowhere? Lancet Oncol. 12: $314-315$

Soriano A and Davis MP (2011). Malignant bowel obstruction: individualized treatment near the end of life. Cleve. Clin. J. Med. 78: 197-206.

Sung JJ, Lau JY, Goh KL and Leung WK (2005). Increasing incidence of colorectal cancer in Asia: implications for screening. Lancet Oncol. 6: 871-876. 
van Hooft JE, Bemelman WA, Oldenburg B, Marinelli AW, et al. (2011). Colonic stenting versus emergency surgery for acute left-sided malignant colonic obstruction: a multicentre randomised trial. Lancet Oncol. 12: 344-352.

Weitz J, Koch M, Debus J, Hohler T, et al. (2005). Colorectal cancer. Lancet 365: 153-165.

Xu AG, Yu ZJ, Jiang B, Wang XY, et al. (2010). Colorectal cancer in Guangdong Province of China: a demographic and anatomic survey. World J. Gastroenterol. 16: 960-965.

Yang Z, Wang L, Kang L, Xiang J, et al. (2011). Clinicopathologic characteristics and outcomes of patients with obstructive colorectal cancer. J. Gastrointest. Surg. 15: 1213-1222.

Zhang J, Yang S, Xie Y, Chen X, et al. (2012). Detection of methylated tissue factor pathway inhibitor 2 and human long DNA in fecal samples of patients with colorectal cancer in China. Cancer Epidemiol. 36: 73-77. 